\title{
Environmental Modeling for Traffic Noise in Urban Area
}

\author{
Francis Cirianni and Giovanni Leonardi \\ DIMET Department, University of Reggio Calabria, Reggio Calabria, Italy \\ Via Graziella, Feo di Vito, 89100, Reggio Calabria, Italy, 1 \\ Telephone 0039 (0)965/875237, Fax 0039 (0)965/875207
}

Received 2012-03-23; Revised 2012-04-20; Accepted 2012-06-18

\begin{abstract}
Traffic noise can be classified among the worst factors in terms of damage to people's health and wellbeing. The trend of noise pollution modeling variable from the smart result of classic regressive models in the performance of many assessment models based on mathematical expressions, genetic algorithms and neural networks (of GRNN type, General Regression Neural Network). A methodological approach for the quantitative analysis of traffic noise in urban settings was proposed in the study. We present an analysis of the acoustic data measured in the city of Villa S. Giovanni (Italy), simultaneous measurement is of noise levels and vehicle flow and composition were done. Different prediction models were compared and a classification for the best assessment tool in the analysis of the equivalent level of noise Leq was given. The results show how the neural network approach provides better performance than the classical solution based on statistical analyses. The GRNN network is best suited to the simulation of the phenomenon seems and for the application in more complex areas, with greater variability in the traffic patterns, such as the case considered.
\end{abstract}

Keywords: Urban noise, traffic management, genetic algorithm, neural networks, GRNN

\section{INTRODUCTION}

Traffic noise is considered one of the most invasive type of noise pollution and often the most intrusive of all and has become an issue of immediate concern for public administrations and authorities. The sources of traffic noise are primarily vehicle engines, exhaust systems, tyre-pavement interaction and aerodynamic friction. Therefore, traffic noise prediction models are essential as supports in the design of roads and highways and in the assessment of existing or envisaged changes in traffic noise conditions (Steele, 2001).

The researches conducted on noise level prediction, in different countries, have led to the development of various models. Suksaard et al. (1999) studied a model to predict the environmental impact of traffic noise based on two vehicles classes. A road traffic noise prediction method for northern European countries was discussed by Bendtsen (1999). A noise prediction model based on Monte-Carlo approach was proposed by Lam and Tam (1998). Li et al. (2002) developed a GIS based road traffic noise prediction model. Pamanikabud and Tansatcha (2003) have also used GIS for analyzing highway traffic noise.

A statistical model to estimate road traffic noise in an urban setting was applied by Calixto et al. (2003). Cirianni and Leonardi (2011) proposed an ANFIS model to estimate the traffic noise in urban condition in an Italian context. An exhaustive discussion on early and recent traffic noise prediction models can be found in the review by Steele (2001).

Cammarata et al. (1995) used a neural network scheme as a substitute for the linear regression of earlier models and comparing the results with some classical regressive models found significant improvements with the use of the neural network. This approach has shown to be particularly interesting and was followed by other authors (El Mallawany et al., 1999; Avsar et al., 2004; Parbat and Nagarnaik, 2008). Gundogdu et al. (2005) and Rahmani et al. (2011) have developed a model by using a genetic algorithm as optimization method.

In these models, vehicles are mostly classified in two groups, heavy and light vehicles, occasionally in three groups, light, medium and heavy vehicles. 
The main objective of this study, which was spurred by an infield campaign to measure traffic noise pollution, is the development of a General Regression Neural Networks (GRNN) approach to assess traffic noise. The approach taken on data led us to classify vehicles in five groups: motor vehicles, light goods vehicles, heavy good vehicles, motorcycles and buses.

A benchmarking of the proposed neural technique is done by application, as we measured the noise levels in the urban area of Villa S. Giovanni (Italy) and compared the results with the output of the neural model and of some traditional regressive models. 2

\section{MATERIALS AND METHODS}

Noise data were recorded at 14 survey sites of Villa S. Giovanni. The city was chosen for its territorial characteristics and its geographic location, being the gateway from Italy to Sicily. Also the area is at the center of the project of the Bridge over the Straits of Messina.

We carried out this survey with the following objectives:

- To measure the equivalent noise levels generated by the traffic circulating in the principal roads

- To estimate the noise level with traditional regressive models and with a neural network approach

- To compare the measured values and the predicted ones, to verify the applicability of this neural technique

The survey sites were chosen on the base of the traffic flow patterns of the cities, setting the sites along the route with the highest crossing flows, on urban roads, with medium to high traffic and therefore with medium speeds.

These 14 survey sites are at a reasonable distance from stop signs and intersections so that the effects of acceleration and deceleration of vehicles can be considered not relevant on the recordings. The noise data recording campaign was led using a Bruel \& Kjar Type 2260 Modular Precision Sound Analyzer. It was located at a point with a distance of $15 \mathrm{~m}$ from the closest traffic flow direction and at a height of $1.2 \mathrm{~m}$ above ground level.

In order to assess the environmental noise, the parameters chosen were: equivalent noise level Leq, percentile levels: L10, L50 and L90, which are the parameters adopted by the main Italian Legislation DPR, 2004 for noise pollution. The measures were carried out during day hours, in an interval of time between 8:00 a.m. and 7:00 p.m. for all the 14 sites, obtaining a set consisting of 154 records.
Vehicles were classified in the following five categories: Motorcycles (M), Motor Vehicles (MV), Light Goods Vehicles (LGV), Heavy Goods Vehicles (HGV) and Buses (B).

Table 1 presents the principal traffic parameters and their ranges.

A correlation analysis was led in order to determine the variables which mainly condition the measured noise levels and to find the most suitable prediction model.

Furthermore, to obtain a model that is able to predict the Leq, it is necessary for the model to (2003):

- Be simple enough so it can be used by all professionals involved in urban planning and traffic management

- Require only data which can be easily obtained for the noise level forecast

- Incorporate accurate results according to the subjective perception of the noise

Among the classical regression models, the following three ones best fit these characteristics Burgess (1977), García and Bernal (1985) and an evolution of the UK Department of Transport - CoRTN model (Cirianni and Leonardi 2011). These models are here briefly described:

\subsection{Burgess Model}

Requires characterizing parameters of the vehicular traffic (veh/h $Q$, percentage of heavy goods vehicles $p$ ) and the half width of the roadway $d$ Eq. 1 :

$\mathrm{M} 1: \mathrm{L}_{\mathrm{eq}}=\alpha_{1} \cdot \log (\mathrm{Q})+\alpha_{2} \cdot \mathrm{p}-\alpha_{3} \cdot \log (\mathrm{d})+\alpha_{4}$

Garcia and Bernal Model Eq. 2:

$\mathrm{M} 2: \mathrm{L}_{\mathrm{eq}}=\alpha_{1} \cdot \log (\mathrm{Q})+\alpha_{2} \cdot \mathrm{p}-\alpha_{3} \cdot \log (2 \mathrm{~d})-\alpha_{4}-\mathrm{V}+\alpha_{5}$

where, the average flow speed $V$ is introduced.

\subsection{An Evolution of the Model CoRTN}

From the CoRTN model, developed from the Department of Transport (UK), the following revised equation for the calculation of Leq is obtained by Cirianni and Leonardi (2011), where the half width of the roadway $d$ is introduced Eq. 3:

$$
\begin{aligned}
& \mathrm{M} 3: \mathrm{L}_{\mathrm{eq}}=\alpha_{1} \cdot \log \mathrm{Q}+\alpha_{2} \cdot \log (\mathrm{V}+40+500 / \mathrm{V}) \\
& +\alpha_{3} \cdot \log (15 / \mathrm{d})+\alpha_{4} \cdot \mathrm{p}+\alpha_{5}
\end{aligned}
$$


Table 1. Principal traffic parameters and their related ranges

\begin{tabular}{lllllll}
\hline $\begin{array}{l}\text { Hourly traffic flow } \\
\text { (vehic/h) }\end{array}$ & $\begin{array}{l}\text { Heavy vehicles } \\
(\%)\end{array}$ & $\begin{array}{l}\text { Light Vehicles } \\
(\%)\end{array}$ & $\begin{array}{l}\text { Buses } \\
(\%)\end{array}$ & $\begin{array}{l}\text { Motorcycles } \\
(\%)\end{array}$ & $\begin{array}{l}\text { Mean traffic speed } \\
(\mathrm{km} / \mathrm{h})\end{array}$ & $\begin{array}{l}\text { Half road width d } \\
(\mathrm{m})\end{array}$ \\
\hline $124-968$ & $0.0-13.98$ & $0.0-11.0$ & $0.0-4.3$ & $0.0-16.1$ & $15.0-40.0$ & $6.00-16.00$ \\
\hline
\end{tabular}
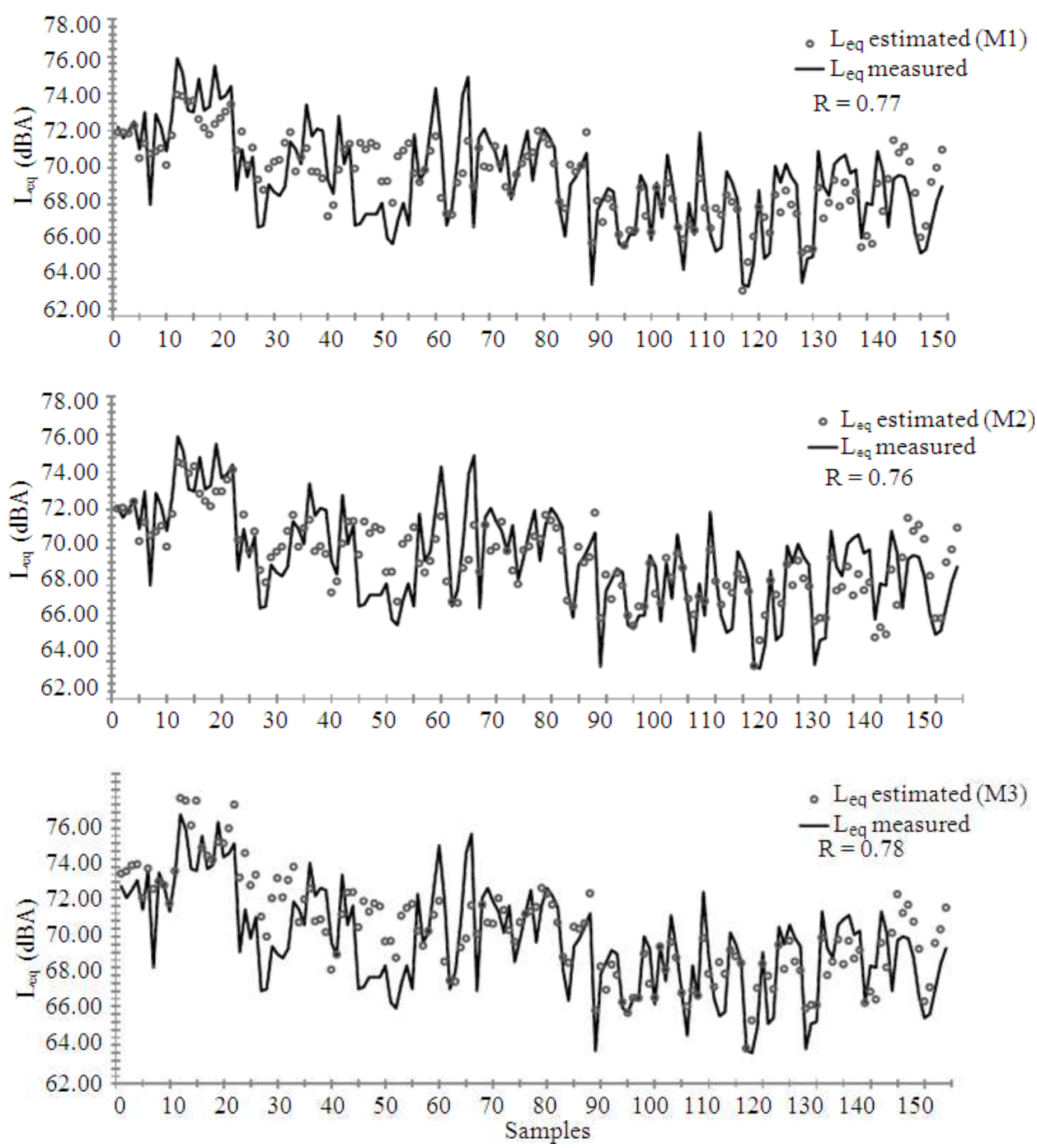

Fig. 1. Graphic comparison $\mathrm{L}_{\mathrm{eq}}$ measured-estimated for the three models

where, the considered variables are: flow $Q(\mathrm{veh} / \mathrm{h})$; average speed $V(\mathrm{~km} / \mathrm{h})$; composition of the vehicular traffic (in terms of percentage of heavy vehicles with an empty weight greater than $1525 \mathrm{~kg}) p(\%)$; slope of the road $G(\%)$.

The models described in the previous paragraph were calibrated and the coefficients of the equations determined by the minimization of the objective function Root Mean Square Error:

$$
\mathrm{f}=\operatorname{RMSE}=\sqrt{\frac{\sum_{\mathrm{i}=1}^{\mathrm{n}}\left(\mathrm{L}_{\mathrm{i}}-\mathrm{L}_{\mathrm{eq}, \mathrm{i}}\right)^{2}}{\mathrm{n}}}
$$

where, $\mathrm{Li}$ and Leq,i are respectively the measured and the calculated noise levels, at time instant $\mathrm{i}$.

Genetic Algorithms (GA) are used in the optimizations. The objective function is multimodal and hence was minimized using genetic algorithms. 
Table 2. Mathematical formulae of the recalibrated models with GA

$\begin{array}{lll}\text { M1: } & \mathrm{L}_{\mathrm{eq}}= & 10838 \cdot \log (\mathrm{Q})+0.127 . \mathrm{p}+40.69 \\ \text { M2: } & \mathrm{L}_{\mathrm{eq}}= & 9565 \cdot \log (\mathrm{Q})+0.166 \cdot \mathrm{p}-0.055 . \mathrm{V}+45.081 \\ \text { M3: } & \mathrm{L}_{\mathrm{eq}}= & 10.213 \log (\mathrm{Q})+6.69 \cdot \log (\mathrm{V}+40+500 / \mathrm{V}) \\ & & -1.544 . \log (15 / \mathrm{d}) 0.103 . \mathrm{p}+44.62\end{array}$

For M1 and M2 the coefficient relative to the distance $\mathrm{d}$ is negligible in regards to the others

Table 3. Input and output variables

\begin{tabular}{lll}
\hline INDICATORS & Variables & \\
\hline Motorcycles & $\mathrm{M}[\mathrm{veh} / \mathrm{h}]$ & INPUT \\
Motor Vehicles & $\mathrm{MV}[\mathrm{veh} / \mathrm{h}]$ & \\
Light Goods Vehicles & $\mathrm{LGV}[\mathrm{veh} / \mathrm{h}]$ & \\
Heavy Goods Vehicles & $\mathrm{HGV}[\mathrm{veh} / \mathrm{h}]$ & \\
Bus & $\mathrm{B}[\mathrm{veh} / \mathrm{h}]$ & \\
Average speed & $\mathrm{V}[\mathrm{km} / \mathrm{h}]$ & \\
Half the width of the track & $\mathrm{d}[\mathrm{m}]$ & OUTPUT \\
Level of acoustic pressure & $\mathrm{L}_{\mathrm{eq}}[\mathrm{dB}(\mathrm{A})]$ & \\
\hline
\end{tabular}

GAs is inspired by Darwin's theory about evolution and are adaptive heuristic search and optimization algorithms based on the principles of genetics and the theory of evolution. GAs is part of the group of Evolutionary Algorithms (EA).

Much of the terminology used in the GA theory borrowed from biology such as genes (orbits), individuals (chromosomes or bit strings) and a population of individuals (structures).

Researchers have successfully applied GAs to solve a variety of optimization engineering, scientific and economic problems that can possess mixed continuous and/or discrete variables, as well as discontinuous and non-convex objective functions.

Classical optimization techniques generate a deterministic sequence of computation based on the gradient or higher-order derivatives of the objective function. This point-to-point approach has the risk of falling in local optima. GAs search among a distributed population of points and use probabilistic rather than deterministic transition rules. Therefore they search more globally and have a good chance to find the global optimum.

A detailed discussion and description of the genetic algorithm theory and application can be found in Mitchell (1998); Goldberg (1989) and Sivanandam and Deepa (2007).

The $\mathrm{a} 1, \mathrm{a} 2, . .$, an parameter of the three equations was determined from the genetic algorithm coded Goldberg's algorithm in MATLAB 2008 setting a population size with a string length of 80 , a crossover probability of 0.001 and a mutation probability of 0.002 .
Using this procedure, the following expressions of the models were obtained.

Figure 1 shows comparisons between the measured Leq values and the calculated ones according to the mathematical models.

Observing Fig. 1, we can see how, even if the correlation coefficient is close to 0.78 for all the models, therefore giving overall a good approximation value, the evaluation quality is still insufficient, as seen in a point to point detail, especially for those values of Leq which greatly differ from the average trend.

To overcome the limits of traditional forecasting approaches, we examined the applicability of a neural approach for traffic noise forecasting and compared the results obtained with the classical regression models previously proposed.

A Neural Network (ANN) can be seen as a system which can answer a question or supply an output in reply to an input and is defined from a certain number of interconnected units of calculation, which operate as a parallel calculation structure and that acquire their knowledge from the experience supplied, that is, the transfer function of the network is not programmed, but is obtained through a process of training with empirical data. In other terms the network learns the function that ties the output with the input by means of the presentation of correct examples of input/output pairs (Specht, 1991; Filho et al., 2004).

Effectively, for every input introduced to the network, in the learning process, the network supplies an output that differs of a given $\delta$ amount from the desired output: the training algorithm modifies some parameters of the network in the desired direction. Every time that an example is introduced, therefore, the algorithm fits slightly the parameters of the network to the values optimal for the solution of the example: in this way the algorithm tries to please all the examples a little at a time.

In order that the comparison be satisfying and complete, it is necessary to consider the great number of analogies with the hypothesis underlying regressive models and neural approach. These analogies essentially consist of the number of variables on which the sound pressure level is made to depend. As illustrated, the independent variables assumed in the regression formulas are generally the vehicular flow, the average speed of the flow, the composition of the flow and, in particular, the percentage of heavy vehicles, defined, according to the Italian standard as the vehicles with an axis load weight exceeding 3.5 tons and the half width of the roadway; these same indicators were used as input data for the achievement of the network. 


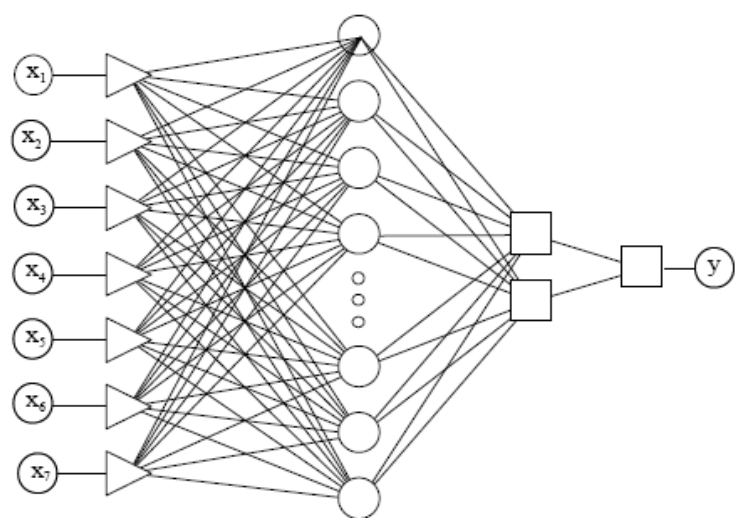

Fig. 2. Neural network of the GRNN family

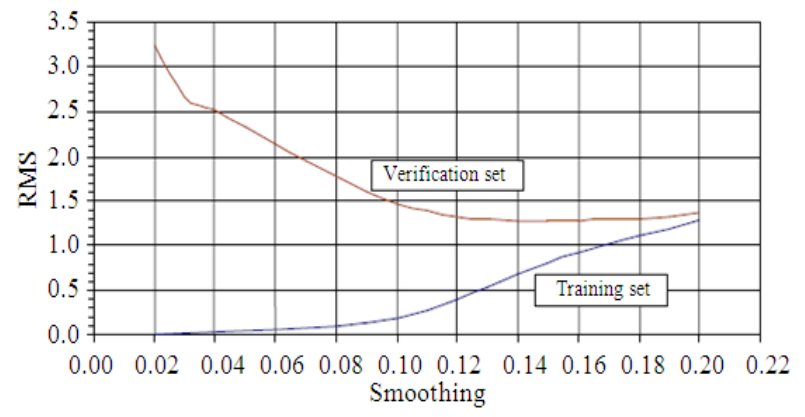

Fig. 3. Trend of the RMSE

So the variables (of input and output) characterizing the neural problem are reported in Table 2 and 3.

The chosen network typology for the best analysis of the problem was the General Regression Neural Network (from here on GRNN). The GRNN approximates any function between input and output and is based on the theory of the not linear regression for the estimation of the function.

The variable regression of one dependent variable $\mathrm{X}$ respect to one independent $Y$ consists in the calculation of the $\mathrm{Y}$ value more probable given $\mathrm{X}$, on the base of a series of pairs $(X, Y)$, subject to experimental error, where $\mathrm{X}$ and $\mathrm{Y}$ can be also victorious. In the field of the identification of systems the dependent variable $Y$ corresponds to the exit of the system corresponding to a given value $\mathrm{X}$ as input.

In order to develop models for the identification of the examined system it is necessary, following a classic regression methodology, to assume a functional shape for the relation between the variables, choosing a set of parameters which fit the experimental data. The values of these parameters are calibrated on the assumed function using the values registered in the field.
The neural network GRNN model doesn't require the predefinition of a functional shape and it defines directing the formulation from the recorded input, independently from any preliminary assumption. In Fig. 2 the scheme of the used GRNN is shown.

The input units supply the network with the elements of vector $X$, vector of which we want to calculate the corresponding expected value. Such values are sent to the neurons of the second layer (pattern units). Every node of the second layer corresponds to an available set and all together constitute a kind of memory of the network.

When a new vector $\mathrm{X}$ is introduced in order to estimate, $\hat{Y}(X)$ its distance from every $X_{i}$ presents in the pattern units is calculated and the result is passed through an exponential function of activation $\exp \left(\mathrm{D}_{\mathrm{i}}^{2} / 2 \mathrm{~h}^{2}\right)$ to obtain a vector of equal dimension to the number of examples available, that constitutes the input for the successive layer (summation units). In correspondence to this layer it is calculated the scalar product between this vector and the weights of the connections and the result is therefore transmitted to the output units which supply the required estimate. The used value of $h$ in the examined case is the one that minimizes the RMSE (root mean squared error) in the verification phase.

Figure 3 shows the trend of RMSE with the variation of the coefficient of smoothing $h$.

The creation of example patterns, which enclose the case type of the chosen data, is the most delicate passage on the way to obtaining reliable results from the neural elaboration.

The available data are grouped by mostly random criteria in three sets: training-set, verification-set and test-set.

The first set, preponderant on the other two, trains the network on the specific problem and for the determination of the weights, the second (verification) estimates the performance and efficiency of the network and definition of the best architecture, lastly, the test data is for the validation of the network once the construction procedure is completed.

\section{RESULTS AND DISCUSSION}

The models (Table 4) are compared on the following statistic parameters: absolute mean error, standard deviation of error, correlation coefficient e Root Mean Square Error (RMSE). 
Table 4. Statistic parameters of models and GRNN neural network

\begin{tabular}{llllll}
\hline Comparison between models and neural network & RMSE & Mean error & Correlation & Standard Deviation \\
\hline GRNN Neural Network & training sets & 0.643 & 0.317 & 0.996 & 0.44 \\
& Verification set & 1.254 & 1.030 & 0.904 & 1.249 \\
& Test sets & 1.361 & 1.121 & 0.874 & 1.478 \\
& All data & 0.941 & 0.514 & 0.938 & 0.928 \\
\hline
\end{tabular}

Recalibrated regressive models

$\begin{array}{lllll}\text { Burgess Model - M1 } & 1.711 & 1.404 & 0.770 & 1.722 \\ \text { Garcia and Bernal Model - M2 } & 1.667 & 1.365 & 0.783 & 1.678 \\ \text { Revised CoRTN model M3 } & 1.855 & 1.494 & 0.767 & 1.731\end{array}$

Fig. 4. Graphic comparison $\mathrm{L}_{\mathrm{eq}}$ measured-estimated by the neural model

Figure 4 shows a comparison between the trends of measured values and values forecast according to the neural models. It can be observed that the considered neural network succeeds in describing the problem adequately, in fact, the estimation error on the output is contained within acceptable values and, moreover, it always guarantees a greater precision than all the other considered regressive models.

This fact allows us to affirm that the GRNN is able to predict satisfactorily the equivalent noise level generated by vehicle flow in roads.

\section{CONCLUSION}

The first aim of the study presented was to define an analysis of different methodologies for the assessment of noise pollution related to road traffic.

The second aim was the development of a traffic noise prediction model that can be used in traffic planning, thus researching traffic noise reduction by redesigning traffic patterns and road layout.

An application with the use of classical regression models was led, opportunely recalibrating the parameters of the models with the aid of genetic algorithms and the results obtained compared with those obtained from a neural network of the GRNN type.

The GRNN model gave the best results in the analysis, also compared to other kinds of neural networks (i.e., the MLP Multilayer Layer Perceptrons network), because:

- The network "learns" in a single step (one pass learning algorithm) and is in a position to generalize as soon as it stores in its "memory" the data available;
- The assessment given is limited by the maximum and minimum values present in the used measurements;

- There are no problems of convergence to solutions corresponding to local minimums of the surface of the errors (as instead happens in the case of Back propagation);

- The version that uses the clustering technique of the data allows the reduction of the calculation time and the development of models that adapt in real time to eventual variations of the systems which they represent.

The results illustrated in the study show how the GRNN network approach is best suited to the simulation of the phenomenon and for the application in more complex areas, with greater variability in the traffic patterns, such as the case considered.

Undoubtedly, taking into account factors such as ground type, classification of vehicles, road surface, reflective surface, would give a more comprehensive model, at the price of more extensive data recording campaigns. However, the results of this study indicate that the neural model can be applied with satisfying results even using the restricted database.

\section{REFERENCES}

Avsar, Y., A. Saral, M.T. Gonullu, E. Arslankaya and U. Kurt, 2004. Neural network modelling of outdoor noise levels in a pilot area. Turkish J. Eng. Environ. Sci., 28: 149-156.

Bendtsen, H., 1999. The Nordic prediction method for road traffic noise. Sci. Total Environ., 235: 331-338. DOI: 10.1016/S0048-9697(99)00216-8

Burgess, M.A., 1977. Noise prediction for urban traffic conditions-related to measurements in the Sydney Metropolitan Area. Applied Acoustics, 10: 1-7. DOI: 10.1016/0003-682X (77)90002-0

Calixto, A., F.B. Diniz and P.H.T. Zannin, 2003. The statistical modeling of road traffic noise in an urban setting. Cities, 20: 23-29. DOI: 10.1016/S0264-2751 (02)00093-8 
Cammarata, G., S. Cavalieri and A. Fichera, 1995. A neural network architecture for noise prediction. Neural Networks, 8: 963-973. DOI: 10.1016/08936080 (95)00016-S

Cirianni, F. and G. Leonardi, 2011. Road traffic noise prediction models in the metropolitan area of the Strait of Messina. Proc. Inst. Civil Eng.-Transport, 4: 231-239.

El Mallawany, A.I., M.I. Abdallah and M.A. El Gawad, 1999. Modeling of traffic noise pollution with neural networks. J. Acoust. Soc. Am., 105: 1335-1335. DOI: $10.1121 / 1.426338$

Filho, J.M.A., A. Lenzi and P.H.T. Zannin, 2004. Effects of traffic composition on road noise: A case study. Transportation Research Part D, Transport Environ., 9: 75-80. DOI: 10.1016/j.trd.2003.08.001

García, A. and D. Bernal, 1985. The prediction of traffic noise levels in urban areas. Proceedings of the International Conference on Noise Control Engineering, (NCE' 85), Munich, Germany, pp: 843-846.

Goldberg, D.E., 1989. Genetic Algorithms in Search, Optimization and Machine Learning. 1st Edn., Addison Wesley Pub Co Inc, ISBN: 0201157675

Gundogdu, O., M. Gokdag and F. Yuksel, 2005. A traffic noise prediction method based on vehicle composition using genetic algorithms. Applied Acoustics, 66: 799-809. DOI: 10.1016/j.apacoust.2004.11.003

Lam, W.H.K. and M.L. Tam, 1998. Reliability analysis of traffic noise estimates in Hong Kong. Trans. Res. Part D: Transport Environ., 3: 239-248. DOI: 10.1016/S1361-9209 (98)00002-9

Li, B., S. Tao, R.W. Dawson, J. Cao and K. Lam, 2002. A GIS based road traffic noise prediction model. Applied Acoustics 63: 679-691. DOI: 10.1016/S0003-682X (01)00066-4
Mitchell, M., 1998. An Introduction to Genetic Algorithms. 1st Edn., MIT Press, Cambridge, ISBN10: 0262631857 , pp: 209.

Pamanikabud, P. and M. Tansatcha, 2003. Geographical information system for traffic noise analysis and forecasting with the appearance of barriers. Environ. Model. Software, 18: 959-973. DOI: 10.1016/S1364-8152 (03)00097-5

Parbat, D.K. and P.B. Nagarnaik, 2008. Artificial neural network modeling of road traffic noise descriptors. Proceedings of the 1st International Conference on Emerging Trends in Engineering and Technology, Jul. 16-18, IEEE Xplore Press, Nagpur, Maharashtra, pp: 1017-1021. DOI: 10.1109/ICETET.2008.220

Rahmani, S., S.M. Mousavi and M.J. Kamali, 2011. Modelling of road-traffic noise with the use of genetic algorithm. Applied Soft Comput., 11: 10081013. DOI: 10.1016/j. Asoc. 2010.01.022

Sivanandam, S.N. and S.N. Deepa, 2007. Introduction to Genetic Algorithms. 1st Edn., Springer, New York, ISBN-10: 354073189X, pp: 442

Specht, D.F., 1991. A general regression neural network. IEEE Trans. Neural Networks, 2: 568-576. DOI: $10.1109 / 72.97934$

Steele, C., 2001. A critical review of some traffic noise prediction models. Applied Acoustics, 62: 271-287. DOI: 10.1016/S0003-682X(00)00030-X

Suksaard, T., P. Sukasem, S.M. Tabucanon, I. Aoi and K. Shirai et al., 1999. Road traffic noise prediction model in Thailand. Applied Acoustics, 58: 123-130. DOI: 10.1016/S0003-682X (98)00069-3 